\title{
Minorías religiosas y centros de culto: percepción social y problemáticas para la gestión pública ${ }^{1}$
}

\section{Luzio Uriarte González}

Universidad de Deusto

luzio.uriarte@deusto.es

\section{Gorka Urrutia Asua}

Instituto de Derechos Humanos, Universidad de Deusto

gorka.urrutia@deusto.es

Orain arte hain ikusgai izan ez bada ere, Euskadiko errealitate sozio-erlijiosoak eraldaketa esanguratsu bat jasan du azkenengo hamarkadetan, askotarikotasun handiagoa gauzatuz. Bere berritasun erlatiboak, besteak beste, zailtasunak dakartza hainbat arlotan eta planotan, bereziki gutxiengo erlijiosoen kasuan eta beraien praktika erlijiosoak jarduteko eskubideak erabiltzean. Lan honetan gertaera honen erradiografia garatu da, bai administrazio publikoaren ikuspuntutik bai erlijio gutxiengoenetik, errealitate honetatik sortzen ari diren erronka nagusiak identifikatuz.

\section{GAKO-HITZAK:}

Aniztasuna, erlijioa, talde gutxituak, herriadministrazioa.
La realidad socio-religiosa del País Vasco ha sufrido una transformación significativa en las últimas décadas, cristalizándose una mayor pluralidad, poco visible hasta la fecha. Su relativa novedad, entre otras cuestiones, acarrea dificultades en diversos planos y ámbitos, especialmente en el caso de las religiones minoritarias y el ejercicio efectivo de su práctica religiosa. Este artículo lleva a cabo una radiografía de este hecho tanto desde la perspectiva de la Administración Pública como desde las propias confesiones minoritarias, identificando los principales desafíos que emergen de esta realidad.

\section{Palabras clave:}

Diversidad, religión, minorías, Administración Local. 


\section{Introducción}

En las últimas décadas, el País Vasco ha sido testigo de una transformación significativa en términos socio-religiosos. En este tiempo se ha dado una caída de la religiosidad (católica) y, por otra parte, se ha ido produciendo una cristalización de una pluralidad religiosa a través de una mayor presencia de otras confesiones. La poca visibilidad de las entidades religiosas no católicas, unida a esa cristalización, ha acarreado ciertas dificultades en diversos ámbitos vinculados al ejercicio efectivo de su práctica religiosa, en especial en las cuestiones relacionadas con la apertura y todo lo relacionado con los centros de culto.

Este artículo aborda estas cuestiones, en primer lugar exponiendo las características más relevantes de las minorías religiosas en la Comunidad Autónoma del País Vasco (CAPV), como ya se ha indicado, una realidad poco conocida. En segundo lugar, a partir de los datos recogidos en el estudio monográfico llevado a cabo por el Gobierno Vasco sobre la diversidad religiosa (Gabinete de Prospección Sociológica, 2012), se exponen los aspectos más relevantes relacionados con la percepción que la sociedad vasca tiene de la diversidad religiosa en el País Vasco, así como su valoración del ejercicio de las prácticas religiosas de las confesiones minoritarias y, en particular, sobre la implantación de centros de culto. En tercer lugar, ahondando en esta cuestión de los centros de culto, se recogen los principales hallazgos obtenidos en el marco de un proyecto de investigación sobre las demandas y necesidades de las entidades religiosas no católicas. Por último, el artículo concluye con una serie de consideraciones sobre la percepción social y la gestión pública de los centros de culto de las religiones minoritarias en el País Vasco.

\section{Entidades religiosas no católicas en la CAPV}

La presencia de confesiones no católicas en el País Vasco ha sido, y sigue siendo, bastante minoritaria y poco visibilizada. Abordar cualquier cuestión relacionada con esta realidad social precisa llevar a cabo previamente una breve presentación de la información disponible sobre su implantación en este territorio, así como una breve referencia a los cambios normativos e institucionales y de la propia sociedad con relación a la religiosidad. Uno de los principales hitos en lo que se refiere a la libertad religiosa, al igual que con relación a cualquier otra libertad, es la aprobación de la Constitución Española de 1978 (CE). La inclusión de la libertad de religión en su articulado, así como el posterior desarrollo en otros instrumentos normativos, supone un punto de inflexión en el reconocimiento institucional de las confesiones no católicas.

Con la promulgación de la Ley Orgánica de Libertad Religiosa (LOLR) en 1980, se dispone de un marco normativo adecuado para la implantación y despliegue de una pluralidad religiosa no existente hasta la fecha. Además, en el caso particular de las religiones minoritarias con mayor arraigo social, y desde una perspectiva histórica con cierta presencia previa (comunidades judía, evangélica e islámica), el año 1992 supone otro punto de inflexión relevante ya que es en esta fecha en la que se rubrican los acuerdos de cooperación entre el Estado y cada una de ellas. Su relevancia reside en el contenido concreto de los acuerdos, así como en el mero hecho de su establecimiento y el potencial marco de relación que a partir de ellos se podía prever entre representantes de las confesiones minoritarias y representantes de la Administración Pública para el pleno desarrollo de las necesidades recogidas en su contenido².

La firma de estos acuerdos es un mero desarrollo de la posibilidad que el artículo 16.3 de la CE ofrece con la posibilidad de establecer formas de relación entre los poderes públicos y las distintas confesiones implantadas en su territorio. Por otra parte, el artículo 7.1 de la LOLR especifica que el Estado podrá materializar acuerdos de cooperación con aquellas confesiones que hayan alcanzado "notorio arraigo". Antes de desarrollar este tipo de acuerdos, un requerimiento es la inscripción en el registro de entidades religiosas, tal y como establece el artículo 5.1 de la LOLR.

Este marco normativo ofrece una variedad de fórmulas en términos de relación o cooperación institucional entre el Estado y las confesiones del que es posible identificar una categorización de las diversas religiones implantadas a nivel estatal. Dejando a un lado el caso particular de la Iglesia Católica, cuyos acuerdos de cooperación con el Estado tienen rango de tratado internacional (por estar firmados con la Santa Sede), entre el resto de confesiones se podrían identificar varias categorías (Díez de Velasco, 2010:246-249; Pérez-Agote, 2012: 45-46). El hecho de formar parte de una o de otra de estas categorías determina, cuando menos formalmente, la posibilidad de desarrollar ciertas disposiciones recogidas en la LOLR.

El primer tipo agrupa aquellas entidades que forman parte de las confesiones que han firmado acuerdos de cooperación con el Estado (previamente han debido recibir el reconocimiento del notorio arraigo). En la actualidad el número de religiones que han formalizado su relación con el Estado en esta modalidad son tres: la comunidad evangélica (por medio de la Federación de Entidades Religiosas Evangélicas de España -FEREDE-), la judía (por medio de la Federación de Comunidades Judías de España -FCJE-), y la musulmana (por medio de la Comisión Islámica de España $-\mathrm{ClE}-)^{3}$. Los acuerdos

${ }^{2}$ Para profundizar en los acuerdos, ver Contreras (2007).

3 El "notorio arraigo" se concedió a las tres confesiones (protestantismo, judaísmo e islam) de una manera genérica en 1989, pero de cara a los acuerdos estas confesiones tuvieron que articularse en federaciones, de tal manera que estas funcionan como estructuras representativas del conjunto de sus comunidades y así, entre otras 
datan del año 1992 (firmados el 28 de abril y ratificados por las leyes 24/1992, 25/1992 y 26/1992 del 10 de noviembre).

Bajo el paraguas de estas confesiones, en la CAPV hay una implantación considerable de comunidades evangélicas y musulmanes, tal y como veremos más adelante. Por el contrario, no hay constancia de una presencia institucional de entidades vinculadas a la comunidad judía. Las comunidades evangélicas e islámicas tienen una triple opción de adhesión a entes federativos. En primer lugar, a través de una iglesia o entidad religiosa de ámbito central y que esté integrada en alguna de las dos entidades federales que forman parte de los acuerdos de cooperación: FEREDE y CIE. En segundo lugar, pueden estar adheridas directamente a alguna de estas dos entidades. Por último, pueden adherirse a alguna entidad federativa de ámbito autonómico que a su vez forme parte de alguna de las dos entidades federales de ámbito estatal: Consejo Evangélico del País Vasco, Unión de Comunidades Islámicas del País Vasco, Federación Islámica del País Vasco y Consejo Islámico de Euskadi), la primera para las iglesias evangélicas y las tres últimas para las comunidades islámicas.

La casuística en el conjunto de estas entidades es variada, ya que pueden estar adheridas a la entidad federativa de ámbito estatal, a alguna entidad federativa de ámbito autonómico, a ambas entidades a la vez, o incluso disponer de ese paraguas federativo a través de la iglesia matriz (en este caso, únicamente las comunidades evangélicas).

El segundo tipo de categoría recoge las confesiones que han alcanzado el notorio arraigo. Este reconocimiento se obtiene a partir de una presencia significativa, tanto en términos cuantitativos de número de fieles como en la implantación territorial ${ }^{4}$. La Comisión Asesora de la Libertad Religiosa del Ministerio de Justicia es el órgano que reconoce el notorio arraigo, si bien es cierto que este reconocimiento no supone aportaciones especiales. Además de las mencionadas tres religiones que tienen firmados acuerdos de cooperación, el Estado ha otorgado este reconocimiento en los últimos años a otras cuatro confesiones: la Iglesia de Jesucristo de los Santos de los Últimos Días (IJSUD) en el año 2003, los Testigos Cristianos de Jehová en 2006, la comunidad budista (Federación de Comunidades Budistas de España -FCBE-) en 2007 y la Iglesia Ortodoxa Cristiana en 2010.

Las confesiones caracterizadas por este reconocimiento podrían clasificarse en dos tipos si nos atenemos a su estructura organizativa. Por un lado, estarían aquellas confesiones caracterizadas

cuestiones, facilitan la interlocución con el Estado.

4 Los tres criterios son la representatividad social, por medio de un número relevante de fieles de su confesión presentes en España; la presencia temporal considerable de forma legal o clandestina; y el ámbito presencial o grado de difusión por medio de lugares de culto o personal religioso. por una iglesia única y de estructura centralizada, a saber, los testigos de Jehová y la Iglesia de Jesucristo de los Santos de los Últimos Días. Ambas confesiones cuentan con un número de registro único para el conjunto de entidades implantadas en el ámbito estatal. Estas iglesias están sumamente organizadas y estructuradas, caracterizadas por su jerarquía y por una sólida articulación entre todas ellas.

Por otro lado, nos encontramos con las confesiones que no tienen una estructura tan centralizada y articulada, como las comunidades budistas y ortodoxas cristianas. En el primer caso, las entidades implantadas en el País Vasco no tienen ningún tipo de articulación de carácter autonómico y, por otra parte, ninguna de ellas está adherida a la Federación de Comunidades Budistas de España (FCBE), que es la entidad federativa de las comunidades budistas a nivel estatal. En el caso de las iglesias ortodoxas, la comunidad ortodoxa conformó su propia Asamblea Episcopal para España y Portugal en el año 2010. La práctica totalidad de comunidades ortodoxas de ámbito local forman parte de dicha asamblea a través de sus respectivos patriarcados. Previamente, algunas iglesias ortodoxas habían recibido "hospitalidad jurídica" por medio de la FEREDE.

En tercer lugar se encuentran las iglesias, confesiones o comunidades de religiones no católicas inscritas en el registro de entidades religiosas (RER). Su inscripción concede personalidad jurídica a aquellas que desean formalizar su situación jurídica como entidad religiosa. Actualmente, a nivel estatal, existen más de $\mathbf{2 . 5 0 0}$ iglesias, confesiones o comunidades religiosas pertenecientes a alguna religión minoritaria. La mayoría de ellas pertenece a alguna de las confesiones con acuerdos de cooperación con el Estado. En último lugar quedarían aquellas confesiones que no están inscritas en el registro de entidades religiosas.

A modo de síntesis, en la Tabla 1 podemos visualizar la categorización de las entidades religiosas vinculadas a alguna confesión minoritaria.

Tabla 1. Categorización de las confesiones minoritarias en el País Vasco a partir de su reconocimiento jurídico (entre paréntesis, año de la firma o reconocimiento)

\begin{tabular}{|l|l|}
\hline Categoría & Entidades con presencia en la CAPV \\
\hline $\begin{array}{l}\text { Confesiones con } \\
\text { acuerdos de cooperación } \\
\text { con el Estado (1992) }\end{array}$ & Evangélicos (FEREDE) \\
\cline { 2 - 2 } $\begin{array}{l}\text { Confesiones que han sido } \\
\text { declaradas de notorio } \\
\text { arraigo }\end{array}$ & IJSUD (2003) \\
\cline { 2 - 2 } \begin{tabular}{l} 
Testigos de Jehová (2006) \\
\cline { 2 - 2 }
\end{tabular} & Budistas (2007) \\
\cline { 2 - 2 } $\begin{array}{l}\text { Confesiones inscritas en } \\
\text { el RER }\end{array}$ & Cristianos ortodoxos (2010) \\
\hline $\begin{array}{l}\text { Confesiones no inscritas } \\
\text { en el RER }\end{array}$ & Varias \\
\hline
\end{tabular}

Fuente: Elaboración propia. 
Para completar la visualización de estas categorías, a partir del grado de federación del conjunto de iglesias, confesiones y comunidades religiosas de las religiones minoritarias en el País Vasco, recogemos en la Tabla 2 el número y porcentaje de las que están adheridas a algún tipo de federación de ámbito estatal. Como se puede observar, el grado de federación o adscripción a alguna iglesia o confesión central tiene un índice relativamente alto, ya que asciende a casi dos tercios del total. Esta adhesión permite a las entidades no católicas una mayor capacidad de relacionarse con la Administración Pública.

Tabla 2. Iglesias, confesiones y comunidades religiosas minoritarias de Euskadi adheridas a federaciones 0 entidades de ámbito estatal

\begin{tabular}{|l|c|c|}
\hline Federación & Total & Porcentaje \\
\hline FEREDE & 96 & $33 \%$ \\
\hline CIE & 18 & $6 \%$ \\
\hline IJSUD- mormones & 4 & $1 \%$ \\
\hline Testigos de Jehová & 65 & $22 \%$ \\
\hline Ortodoxos & 9 & $3 \%$ \\
\hline No federadas & 103 & $35 \%$ \\
\hline TOTAL & 295 & $100 \%$ \\
\hline
\end{tabular}

Fuente: Urrutia Asua (2016).
La información de los datos reflejados en la tabla anterior muestra un alto grado de asentamiento e institucionalización de la mayoría de las entidades y comunidades religiosas no católicas. Además del hecho de estar inscritas en el Registro de Entidades Religiosas del Ministerio de Justicia, gran parte de ellas están adheridas a alguna entidad federativa, hecho que les da un mayor grado de institucionalización. Estos dos elementos son reflejo de esa búsqueda de reconocimiento formal, así como de una voluntad de disponer de espacios de interlocución de carácter más formal.

Esta voluntad ya mencionada se encuentra alineada con el tipo de cooperación que promulga la Constitución Española a partir del artículo 16.3, que ilustra el Estado español como un Estado aconfesional caracterizado por ser un modelo de carácter colaborativo entre el Estado y la Iglesia católica, así como el resto de las confesiones. Sin embargo, esto no se suele visualizar con suficiente claridad, sobre todo en ciertos ámbitos, como el local.

En añadidura a la información disponible sobre el total del número de entidades religiosas no católicas en la CAPV, un dato de interés es el de la evolución del número de estas entidades en Euskadi a lo largo de las últimas décadas. Como podemos observar en el Gráfico 1, la creación de entidades religiosas no católicas ha tenido un crecimiento constante hasta la actualidad.

En definitiva, el total de entidades religiosas no católicas es de 295 comunidades vinculadas a diferentes religiones, la mayoría de ellas con el reconocimiento del notorio arraigo.

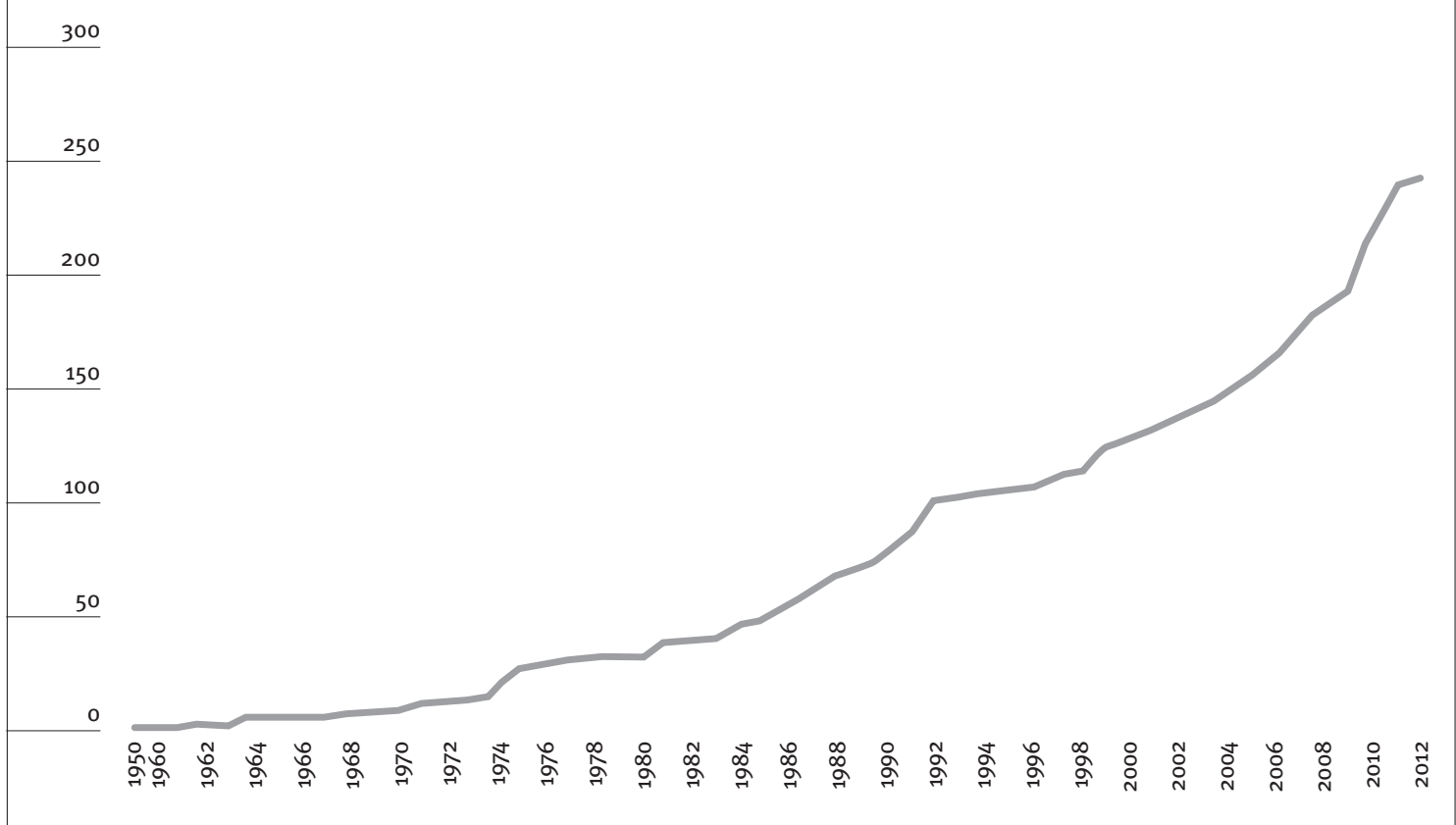

Fuente: Elaboración propia a partir de la base de datos (fecha corte: 29/06/2013). 


\section{Percepción social y valoración de la diversidad religiosa en la opinión pública vasca}

En mayo de 2012 el Gabinete de Prospecciones Sociológicas del Gobierno Vasco llevó a cabo un estudio monográfico sobre la diversidad religiosa en el País Vasco. Aprovechamos parte de la información recogida en ese estudio para ofrecer un análisis complementario a la información disponible de nuestro estudio con el fin de poder ofrecer una panorámica más completa.

Así, comenzando por la percepción que se tiene de la realidad sociorreligiosa, en la Tabla 3 se puede observar cierto grado de distorsión entre lo percibido y la autoubicación en cuanto a la composición religiosa. La percepción mayoritaria alcanza el $71 \%$ para las personas católicas no practicantes, mientras que la autoubicación es del $57 \%$ según datos del estudio del Gabinete de Prospección Sociológica del Gobierno Vasco. Las diferencias son también notables para el resto de opciones (católicas practicantes, 15 puntos; agnósticas-ateas-no creyentes, 16 puntos; y creyentes de otras religiones, 7 puntos).

A partir de la evolución en los datos de autoidentificación religiosa de la población de la CAPV, se podría afirmar que se está dando un proceso de secularización fuerte; sin embargo, la imagen que proyecta la información de la tabla anterior refleja una percepción de la sociedad como eminentemente católica. Esta brecha entre la identificación y lo percibido manifiesta cierto grado de distorsión y un escaso conocimiento de estos cambios sociorreligiosos.
En cualquier caso, la percepción generalizada sobre la composición religiosa de la sociedad vasca es mayoritariamente favorable para la religión católica ( $82 \%$ ) aunque varía la percepción sentida de la autoubicación, sobre todo cuando se matizan las posiciones "practicantes" y las "no practicantes". La diferencia se incrementa sintomáticamente a la hora de estimar el número de personas "agnósticas, ateas o no creyentes", que en el caso de la autoubicación es muy superior a la percepción sentida.

En este sentido, ahondando en la cuestión de la percepción de las confesiones no católicas, podemos observar que, ante el planteamiento de la valoración de su presencia, se podrían constatar tres actitudes o grupos bien definidos (con diferente peso porcentual). En primer lugar, la posición mayoritaria es la de las personas escépticas (51\%) y que no valoran ni bien ni mal la presencia de estas confesiones. En segundo lugar, encontramos a las personas tolerantes (38\%), que son quienes valoran bien o muy bien la presencia de confesiones no católicas. Por último, estaría el sector más crítico, un $8 \%$ de la población al que le parece mal o muy mal esta presencia. Observando los datos a partir de los grupos de edad, es interesante resaltar que el segmento más joven (entre 18 y 29 años de edad) es el que expresa una valoración más positiva.

Otra información de interés para valorar la presencia de las confesiones minoritarias en Euskadi es la percepción existente sobre las facilidades para prácticas religiosas de las confesiones no católicas, recogida en la Tabla 5 . Se puede observar que la percepción sobre el grado de facilidad que encuentran las entidades religiosas minoritarias

Tabla 3. Percepción sobre la composición religiosa en Euskadi

\begin{tabular}{|c|c|c|c|c|c|}
\hline \multirow{2}{*}{ Personas } & \multirow{2}{*}{ Totales } & \multicolumn{4}{|c|}{ Grupos de edad } \\
\hline & & $18-29$ & $30-45$ & $46-64$ & $>=65$ \\
\hline Católicas practicantes & 11 & 6 & 8 & 12 & 18 \\
\hline Católicas no practicantes & 71 & 72 & 75 & 74 & 63 \\
\hline Otras religiones & 1 & 0 & 1 & 1 & 0 \\
\hline Agnósticas, ateas o no creyentes & 9 & 14 & 9 & 6 & 5 \\
\hline No sabe/No contesta & 8 & 8 & 7 & 7 & 14 \\
\hline Total & 100 & 100 & 100 & 100 & 100 \\
\hline
\end{tabular}

Fuente: Elaboración propia a partir del estudio de Gabinete de Prospección Sociológica (2012).

Tabla 4. Valoración de la presencia de confesiones no católicas en Euskadi

\begin{tabular}{|l|c|c|c|c|c|}
\hline \multirow{2}{*}{} & \multirow{2}{*}{ Totales } & \multicolumn{4}{c|}{ Grupos de edad } \\
\cline { 3 - 7 } & & $\mathbf{1 8 - 2 9}$ & $\mathbf{3 0 - 4 5}$ & $\mathbf{4 6 - 6 4}$ & $\boldsymbol{\gamma}=65$ \\
\hline Muy mal & 2 & $\mathbf{1}$ & $\mathbf{2}$ & $\mathbf{2}$ & 3 \\
\hline Mal & 6 & 4 & 5 & 5 & 10 \\
\hline Ni bien ni mal & 51 & 49 & 52 & 53 & 48 \\
\hline Bien & 33 & 38 & 33 & 33 & 28 \\
\hline Muy bien & 5 & 8 & 6 & 3 & 3 \\
\hline No sabe/No contesta & 4 & 0 & 2 & 4 & 8 \\
\hline Total & 101 & 100 & 100 & 100 & 100 \\
\hline
\end{tabular}

Fuente: Elaboración propia a partir del estudio de Gabinete de Prospección Sociológica (2012). 
para llevar a cabo sus prácticas religiosas no es muy positiva. En primer lugar, es necesario indicar que se constata un gran desconocimiento sobre esta cuestión, ya que prácticamente una cuarta parte de la población no sabe o no contesta cuando se le pregunta sobre ello. La opinión dominante es la que considera que existen "bastantes o muchas facilidades" ( $43 \%$ ) frente a la que mantiene que las confesiones minoritarias tienen "pocas o ninguna facilidad” ( $33 \%$ ) para sus prácticas religiosas. En este caso, la edad no parece influir en el mismo grado que en los indicadores anteriores, ya que los distintos segmentos se comportan de forma parecida ante las diferentes opciones consultadas.

Los indicadores que hacen referencia al conocimiento y valoración de su presencia social, así como a la percepción sobre las facilidades que encuentran para sus prácticas religiosas, ofrecen una imagen ambigua en lo que se refiere a las confesiones religiosas no católicas. Especialmente, es sintomático el alto grado de desconocimiento expresado sobre esta realidad

Un elemento con un componente de proyección pública relevante y bien significativo es el de los centros de culto. Quizás derivado de esta proyección pública, suscita las posiciones más contradictorias e incluso radicalizadas. La tensión entre la práctica privada de la religión y la pública queda reflejada con bastante claridad en esta cuestión. Así, en la esfera privada y en términos generales, se constata una aceptación de este derecho. Sin embargo, cuando este derecho tiene una proyección pública, como es el caso de la implantación de centros de culto, se incrementan las reticencias 0 , directamente, el rechazo. En las Tablas 6 y 7 podemos observarlo, con los significativos matices que surgen a propósito del reconocimiento del derecho a la apertura de los lugares de culto. Un buen porcentaje de la población considera que todas las religiones tienen derecho a tener e instalar un centro de culto; sin embargo, se constata una restricción de este derecho a "algunas religiones" ( $11 \%$ ) y un rechazo total a tal derecho, para cualquier religión, por parte de un $9 \%$ de la población.

La Tabla 7 nos muestra que la población no es partidaria de facilitad el derecho a instalar centros de culto a ciertas religiones. Esta restricción se focaliza especialmente en el caso de una religión, el islam, y es curioso cómo este rechazo es generalizado entre todas las franjas de edad.

Tabla 5. Percepción sobre las facilidades para prácticas religiosas de las confesiones no católicas en Euskadi

\begin{tabular}{|l|c|c|c|c|c|}
\hline & \multirow{2}{*}{ Totales } & \multicolumn{5}{|c|}{ Grupos de edad } \\
\cline { 3 - 7 } & & $\mathbf{1 8 - 2 9}$ & $\mathbf{3 0 - 4 5}$ & $\mathbf{4 6 - 6 4}$ & $\boldsymbol{\gamma}=\mathbf{6 5}$ \\
\hline Ninguna facilidad & 3 & 3 & 2 & 3 & 4 \\
\hline Pocas facilidades & 30 & 35 & 28 & 30 & 27 \\
\hline Bastantes facilidades & 33 & 38 & 35 & 31 & 27 \\
\hline Muchas facilidades & 10 & 8 & 14 & 11 & 7 \\
\hline No sabe/No contesta & 24 & 16 & $\mathbf{2 1}$ & 25 & 35 \\
\hline Total & 100 & 100 & 100 & 100 & 100 \\
\hline
\end{tabular}

Fuente: Elaboración propia a partir del estudio de Gabinete de Prospección Sociológica (2012).

Tabla 6. Derecho a tener e instalar centros de culto

\begin{tabular}{|c|c|c|c|c|c|c|c|}
\hline & \multirow{2}{*}{ Totales } & \multicolumn{4}{|c|}{ Grupos de edad } & \multicolumn{2}{|c|}{ Relación con otras religiones } \\
\hline & & $18-29$ & $30-45$ & $46-64$ & $>=65$ & Si & No \\
\hline Sí, todas las religiones & 73 & 82 & 73 & 73 & 64 & 78 & 69 \\
\hline Algunas religiones sí y otras no & 11 & 8 & 11 & 11 & 12 & 11 & 10 \\
\hline No, ninguna religión & 9 & 6 & 10 & 10 & 11 & 6 & 13 \\
\hline No sabe/No contesta & 7 & 4 & 6 & 6 & 13 & 5 & 7 \\
\hline Total & 100 & 100 & 100 & 100 & 100 & 100 & 100 \\
\hline
\end{tabular}

Fuente: Elaboración propia a partir del estudio de Gabinete de Prospección Sociológica (2012).

Tabla 7. Religiones a las que no debería autorizarse la apertura de centros de culto

\begin{tabular}{|c|c|c|c|c|c|}
\hline & \multirow{2}{*}{ Totales } & \multicolumn{4}{|c|}{ Grupos de edad } \\
\hline & & $18-29$ & $30-45$ & $46-64$ & $>=65$ \\
\hline Islam & 49 & 48 & 46 & 59 & 44 \\
\hline Evangélicos/Protestantes & 4 & 2 & 5 & 5 & 3 \\
\hline Testigos de Jehová & 2 & 4 & 2 & 1 & 3 \\
\hline Sectas & 2 & 4 & 3 & 0 & 0 \\
\hline Otras & 8 & 10 & 8 & 7 & 8 \\
\hline No sabe/No contesta & 39 & 34 & 38 & 38 & 43 \\
\hline Total & 104 & 102 & 102 & 110 & 101 \\
\hline
\end{tabular}

Fuente: Elaboración propia a partir del estudio de Gabinete de Prospección Sociológica (2012). Los porcentajes no suman 100 porque se podían dar dos respuestas. 
Para completar la información sobre la percepción de la diversidad religiosa en Euskadi, el grado de molestia ante la cercanía de los lugares de culto de las diferentes religiones nos permite calibrar el grado de tolerancia de la población vasca ante la presencia de creyentes o entidades religiosas minoritarias. Este mismo estudio dispone de esta información, que recogemos brevemente en las siguientes líneas.

El grado de malestar no es alto, ya que a la mayoría de la población no le molesta o le molesta poco tener un centro de culto próximo a su lugar de residencia (entre el $96 \%$ para los centros católicos y el $71 \%$ para los musulmanes). A partir de la información disponible es posible establecer tres bloques de aceptación "territorial" de centros de culto: a) de muy alta aceptación, el correspondiente a los centros católicos y de otras religiones (más del $91 \%$ de aceptación o no molestia); b) de alta aceptación (entre $80 \%$ y $90 \%$ ), ortodoxos, testigos de Jehová y evangélicos; c) de relativa aceptación (71\%), musulmanes. En este caso se manifiesta además el rechazo más elevado de todas las confesiones: a casi una de cada cuatro personas ( $23 \%$ ) le molestaría mucho o bastante la cercanía de un centro de culto musulmán.

Las reticencias aumentan con la edad para el caso de los testigos de Jehová y de los musulmanes. Los grupos de más edad experimentan un mayor rechazo o malestar a la cercanía de centros de culto a sus domicilios, de los testigos y de los musulmanes (con oscilaciones de 10 puntos entre el grupo de los “jóvenes" de 18-29 años y el grupo de mayores de 65 años).

\section{Gestión pública de la diversidad religiosa y centros de culto: principales problemáticas}

Como hemos podido observar hasta aquí, las entidades religiosas no católicas en la CAPV tienen un grado de implantación significativo en términos de temporalidad y en cuanto al número de comunidades religiosas. Esta situación es muy similar a la que se da en el ámbito estatal, y al igual que en otros lugares las necesidad o demandas que plantean los representantes de estas religiones minoritarias son bien similares. Estas demandas abarcan diferentes cuestiones, pero de entre todas ellas nos centraremos en las que tienen que ver con los centros de culto, ya que, tal y como hemos podido observar en el apartado anterior, se trata de uno de los elementos más polémicos, y sin embargo, de necesidad primordial para el ejercicio de la libertad religiosa.

Antes de proseguir, destacamos la relevancia que desde una perspectiva normativa tienen los centros de culto. Así, este es un derecho recogido en el desarrollo que de la libertad de religión se hace en la Ley Orgánica de Libertad Religiosa, concretamente en su artículo $2^{5}$. Este articulado se ve reforzado, en el caso de las confesiones con acuerdos de cooperación con el Estado (comunidades judía, evangélica y musulmana), también en el artículo segundo de los tres acuerdos, ya que recogen este derecho en su articulado.

En cualquier caso, más allá de los aspectos meramente normativos, disponer de un espacio físico es una condición muy importante para poder desarrollar la vida comunitaria básica ya que es el elemento que permite el encuentro entre los miembros de estos grupos. Esto posibilita la interacción habitual del grupo y la ausencia de este espacio, perjudica seriamente el dinamismo interno de la comunidad. Hemos de tener en cuenta que la mayor parte de las entidades religiosas se reúnen también como comunidades litúrgicas en las que la celebración comunitaria es parte esencial. Además, este espacio trasciende el dinamismo interno, ya que además de su importancia como espacio físico de reunión, también facilita y posibilita el encuentro y la interacción con el entorno social. La ubicación física de un local visibiliza para una sociedad la existencia de un determinado grupo religioso y posibilita una forma de interacción social muy significativa. El encuentro "en vivo y en directo" facilita un conocimiento y un reconocimiento que es clave en las relaciones sociales y en la generación de confianzas.

En este contexto, es muy comprensible que los grupos religiosos consideren este tema del espacio físico como muy importante para la supervivencia y desarrollo de la comunidad, por lo que de forma natural y espontánea se hace presente en todas las entrevistas con sus representantes. Se trata de un tema muy sensible en el que las posturas críticas crecen en la medida que encuentran dificultades y restricciones a sus aspiraciones de contar con lugares de encuentro.

Aunque disponer de un espacio es importante para todos los grupos, el posicionamiento y la percepción que tienen respecto a las condiciones de acceso a locales están claramente diferenciados. La posición más crítica la encontramos entre los grupos musulmanes y entre los cristianos evangélicos. La comunidad cristiana ortodoxa también expresa sus dificultades, aunque de una forma más atenuada y sin un carácter marcadamente reivindicativo.

${ }^{5}$ Artículo 2 de la Ley Orgánica de Libertad Religiosa:

"Dos. Asimismo comprende el derecho de las Iglesias, Confesiones y Comunidades religiosas a establecer lugares de culto o de reunión con fines religiosos, a designar y formar a sus ministros, a divulgar y propagar su propio credo, y a mantener relaciones con sus propias organizaciones o con otras confesiones religiosas, sea en territorio nacional o en el extranjero.

Tres. Para la aplicación real y efectiva de estos derechos, los poderes públicos adoptarán las medidas necesarias para facilitar la asistencia religiosa en los establecimientos públicos, militares, hospitalarios, asistenciales, penitenciarios y otros bajo su dependencia, así como la formación religiosa en centros docentes públicos".

Para ver el papel de los medios de comunicación con relación a estos temas, ver GARCÍA y URIARTE (2011). 
Los testigos de Jehová trasmiten una posición radicalmente diferente a la del resto, en cuanto que muestran una aceptación y conformidad con el marco legal y su desarrollo, al que se sienten plenamente adaptados y del cual tratan de obtener el mayor provecho posible. De entre todas las cuestiones planteadas por representantes de las confesiones minoritarias, en las siguientes páginas destacamos los núcleos más problemáticos ${ }^{6}$.

\subsection{Requerimientos legales y aplicaciones de criterios para abrir un local de culto}

Disponer de un local para la celebración, el encuentro, la formación o cualquier otro tipo de fin relacionado con sus tradiciones es fundamental para cualquier grupo religioso. Abrir un local de estas características requiere una regularización y formalización ante la Administración Pública. La normativa con la que se encuentran para llevarla a cabo es poco clara y abierta a múltiples interpretaciones ${ }^{7}$. Esta visión es compartida tanto por las personas entrevistadas de los grupos religiosos como por parte de los técnicos municipales, estos últimos indican incluso que la normativa que regula la apertura de locales religiosos o bien no existe específicamente o bien es deficiente para ser aplicada directamente. En ocasiones, como la que recogemos en las siguientes líneas, los gobiernos municipales que se encuentran por primera vez con una situación en la que se solicita la apertura de un local de estas características expresan su sorpresa ante este hecho: "[...] nos han solicitado [...] la posibilidad de instalar alguna mezquita, centro de culto, una cosa así; esto es nuevo para nosotros, primero, porque nunca hemos tenido esta situación a lo largo de la historia y, segundo, por la problemática, entre comillas, que puede estar surgiendo, por ejemplo en Bilbao, por la instalación de una mezquita y estamos dándole vueltas a la situación”.

Esa sorpresa se entiende en un contexto en el que no existe ninguna normativa específica para este tipo de equipamientos, lo cual hace que, subsidiariamente, se suelan aplicar unos requerimientos legales pensados para otros tipos de actividades, con los problemas que ello encierra, como por ejemplo la toma de decisiones arbitrarias que los grupos minoritarios no comprendan fácilmente: “[...] vemos en este momento la normativa que regula la apertura de las mezquitas es bastante defectuosa, por no

6 Para profundizar en estas cuestiones ver MORONDO TARAMUNDI, D. y RUIZ VIEYTEZ, E.J. (eds.) (2014): Diversidad religiosa, integración social y acomodos. Un análisis desde la realidad local en el caso vasco, Bruselas, Peter Lang.

7 Una presentación del marco jurídico general para el caso español y de su desarrollo normativo se puede consultar en PONCE J. y CABANILLAS, J.A., 2001. Al respecto, son también significativas las declaraciones de Mariano Bláquez, secretario ejecutivo de la FEREDE, denunciando la falta de una normativa específica que regule la apertura de los lugares de culto (cfr. http://www. observatorioreligion.es/entrevistas/mariano_blazquez/index.html, consultado el 20/10/2014). decir inexistente, se aplica de manera subsidiaria la norma relativa a la apertura de locales de negocio para un centro de culto, lo cual tampoco nos parece a nosotros lo más adecuado, empezando ya con la regulación jurídica defectuosa, que no nos extrañe que luego vayan a derivarse de ahí otras series de problemas o de cuestiones".

En el siguiente extracto de una entrevista realizada a un miembro de una iglesia evangélica, podemos observar el malestar que ese tipo de situaciones genera: “La Ley de locales de culto va a ser equiparar un local de culto que tienen un par de reuniones a la semana, que cantas las canciones, con un nivel de insonorización de una discoteca, cuando se exigen salidas de emergencia, baños adaptados. Cuando tú vas a cualquier Iglesia Católica, y bueno..."

Como ya hemos indicado, el marco legal para la gestión de apertura y funcionamiento de los locales de grupos religiosos es poco claro, lo que se refleja en diversas manifestaciones de los propios técnicos municipales: "vemos en este momento la normativa que regula la apertura de mezquitas es bastante defectuosa, por no decir, inexistente". "Yo creo que se está dejando hacer, se está mirando para otro lado".

Este contexto da lugar a una heterogeneidad de criterios y de formas de actuación frente al requerimiento de locales por parte de las minorías religiosas. El mantenimiento de esta ambigüedad conlleva varias consecuencias negativas. Por una parte, algunos grupos se pueden sentir discriminados respecto a los criterios que la autoridad aplica a otros. Esta sospecha aparece especialmente, aunque no solo, en el caso de la comunidad musulmana. La arbitrariedad no favorece la transparencia de la gestión pública de este tema y añade una cierta desconfianza y rivalidad entre los diferentes grupos religiosos. Por otra parte, los gobiernos municipales también pueden entrar en competencia entre sí, tratando de no asumir responsabilidades y de desviar hacia otros municipios la apertura de nuevos locales y centros de culto. Es por ello por lo que es necesario abordar la cuestión de la regulación y planificación de estas cuestiones desde una perspectiva más global.

\subsection{Regulación y planificación municipal del suelo}

En este contexto de cierta ambigüedad sobre el marco que regula la apertura y el uso de los locales religiosos entra la posibilidad de que los municipios dicten normas de regulación del suelo para la instalación de nuevos grupos religiosos en diferentes lugares. Esta fue la situación que se dio en el municipio de Bilbao en el año 2013, cuando su Ayuntamiento aprobó una norma con el fin de regular el uso de espacios dentro de la ciudad para fines religiosos, mediante la modificación de su Plan General de Ordenación Urbana. Esta norma contemplaba la restricción de la apertura de centros de culto en edificios residenciales de la ciudad, de 
manera que las confesiones minoritarias asentadas en el municipio de Bilbao veían cómo se reducía sustancialmente el espacio físico en el que poder implantar centros de culto ${ }^{8}$.

En este marco, son varias las muestras del personal técnico de ámbito municipal en las que se puede observar la necesidad o la expectativa sobre la elaboración de un marco general respecto a la diversidad religiosa y en el que se incluyan criterios para regular, entre otros temas, el acceso a los locales de culto.

“[...] creo que hay que ser valientes en este caso; que se establezca una normativa desde el punto de vista autonómico que regule a todos, porque al final si haces una normativa o una ordenanza municipal, tiene el valor que tiene dentro del municipio y eso puede generar lo que los alcaldes no quieren, el efecto llamada".

“[...] sí que tienen un espacio y, por lo que me trasmitieron los representantes de la asociación, se les queda pequeño dadas las necesidades que tienen; lo que querían era buscar otro local que ya tenían identificado pero para saber si realmente había posibilidades de legalizarlo, estamos un poco a la espera del tema del borrador del Gobierno Vasco".

Aunque los gobiernos municipales tengan buena intención a la hora de gestionar la diversidad religiosa y ofrecer oportunidades justas para todos los grupos, tal y como podemos observar en esta última cita, parece conveniente plantear una política global en el territorio, más allá de las iniciativas concretas que pueda tener una determinada localidad. En este sentido, tiene su lógica abordar esta cuestión desde una panorámica global, ya que se pueden producir desequilibrios territoriales y sociales, así como tensiones que no ayuden a la convivencia y que sean difíciles de encauzar en el futuro. En un caso extremo, podría derivar en una segregación territorial socialmente muy poco aconsejable.

En efecto, esta es una problemática añadida, ya que si contar con un local propio es de especial relevancia para la vida del grupo, su inserción en el entorno también juega un papel muy importante. La localización dentro del espacio urbano, en términos de accesibilidad y cercanía a los lugares de residencia de sus miembros, incide directamente en su participación. De la misma manera, todo ello facilita la interacción con otros grupos sociales y el vecindario en general. En los casos contrarios, si el local está ubicado en zonas poco accesibles (como los extrarradios o en zonas que no constituyen

${ }^{8}$ Más información sobre este tema se puede consultar en RUIZ VIEYTEZ, E.J; (2013): “Derechos humanos, discriminación y diversidad religiosa en el País Vasco: la polémica sobre la apertura de centros de culto en Bilbao"; en MORENO, G. (ed.); Anuario Ikuspegi 2013, Bilbao, Ikuspegi, 2013, pp. 283-294. lugares de paso habituales), se dificultan las funciones que debe cumplir un espacio de estas características.

En este sentido, algunos de los grupos religiosos perciben cierta intencionalidad por parte de las autoridades a la hora de facilitar la apertura y ubicación de centros de culto. Esto, obviamente, también incide en la visibilidad social de la comunidad.
"[...] hay instituciones vascas que quieren promover que las mezquitas se encuentren en lugares donde puedan tener un terreno, digamos, con un entorno segregado, tanto para que por un lado puedan tener más medios esas mezquitas como para que el impacto que pueda hacer sobre el entorno en momentos puntuales, la presencia de un gran número de personas se amortigüe, y sobre todo es el punto de vista de que son personas de diferentes orígenes”.

Esta persona, perteneciente a uno de los colectivos que peor percibidos (islam), expresa la convicción de que existe una intencionalidad por parte de algunas instituciones para que las mezquitas o los lugares de oración musulmana no se sitúen en el centro de la ciudad. Una de esas razones está relacionada con la finalidad de evitar molestias a los vecinos, y es por ello por lo que en algunos lugares se propone que determinados centros de culto sean desplazados a zonas industriales, tal y como lo expresa el siguiente representante municipal.

\section{“[...] hemos intentado atacar este problema mediante una permuta de locales para que pudieran ir y montar una mezquita en una zona, en un edificio vacío y además industrial”.}

Este motivo hay que interpretarlo en función de otro que se formula habitualmente, a saber, que las personas que se reúnen en torno a la mezquita son mayoritariamente de origen extranjero, y cuando esto se da con un grupo numeroso puede ser percibido por parte de la ciudadanía con temor, incomodidad o como una cierta amenaza. Es a partir de esta cuestión como se puede interpretar la posición de la persona entrevistada y el hecho de derivar a zonas industriales la implantación de centros de culto, de tal manera que se produce una invisibilidad. En este caso, desde la perspectiva de la persona entrevistada, la segregación espacial tiene un interés por producir la invisibilidad del grupo religioso en la percepción de que puede ser potencialmente conflicto para la sociedad. Esta segregación espacial está agravada con la sospecha de que hay una discriminación negativa respecto a la confesión musulmana en particular. Esta idea emerge por lo menos en la entrevista realizada a un técnico municipal:

"Yo creo que se está dejando hacer, se está mirando para otro lado, se está dejando hacer mientras no sean musulmanes, es decir, cuántas 
lonjas en muy precarias condiciones de los evangelistas hay en muchísimos barrios [...] un montón. Con aglomeración de gente, con ruidos de micrófonos, con más ruido incluso que lo que pueden meter los musulmanes, hay una clara estrategia de señalar con el dedo e impedir los espacios de culto musulmanes, no así los evangelistas y hay muchísimos más".

Se puede observar, por tanto, la preocupación de que la comunidad musulmana se encuentra en clara inferioridad de condiciones cuando trata de reunirse. Por una parte, encontramos una falta de arraigo en un segmento de sus miembros (por las dificultades derivadas con la competencia lingüística y el suficiente conocimiento de costumbres locales) $y$, por otra, la escasez de recursos conlleva cierta tendencia a situarse en espacios marginales de los núcleos urbanos, con la consiguiente marginación en lo que se refiere a las relaciones sociales. Es preciso indicar que, si bien esta cuestión aparece en varias entrevistas, no se trata de una situación generalizada. Más bien se trata de resaltar una preocupación por la inclusión social de estos colectivos de tal manera que la interacción entre grupos se sitúe dentro de cierta normalidad, reconociendo en positivo la diversidad religiosa.

Esta segregación espacial no se produce únicamente con las comunidades musulmanas, ya que otros grupos religiosos también la sufren, dificultando la vida y el encuentro de la comunidad. La comunidad cristiana ortodoxa, por ejemplo, también se encuentra en una misma situación. La diferencia es que en este caso, gracias a un convenio con la Iglesia católica, tienen el acceso a lugares de culto católicos en el entorno de Bilbao y Lasarte.

\subsection{Espacios insuficientes}

Por último, además de la ubicación de los centros de culto, otro aspecto relevante es el de las condiciones internas de los locales en los que se juntan las comunidades religiosas, como por ejemplo el espacio, que permitirá un mayor o menor aforo. La escasez de recursos económicos de estas comunidades les dificulta acceder a locales con suficiente espacio para albergar a todos sus miembros. En algunos casos, además, con requerimientos particulares, como los musulmanes:

“[...] hay tres (locales) en Getxo, en Bilbao y en Barakaldo; también estos centros que hay, o estas mezquitas, en Bilbao o Barakaldo no es suficiente, no es grande para ir mujeres [...], no es como otros países [...], aquí falta mucho; Getxo, que es un barrio muy grande, tiene que tener una para la gente porque hay muchas familias árabes que viven aquí y necesitan, y además tenemos fiestas religiosas".

Además de esta cuestión de la insuficiencia del espacio de locales, la persona entrevistada expone la dificultad de cumplir con la tradición de separar los espacios según el género. Esta misma preocupación por disponer de un espacio suficiente se manifiesta desde la Administración, sin embargo en este caso más bien por cuestiones relacionadas con la seguridad de quienes se reúnen.

“La tramitación que iniciaron (la comunidad musulmana) fue una cosa muy pacífica y muy normal, y lo único que nosotros les pedíamos, sobre todo a nivel administrativo, era el tema de aforo, que era lo que más nos preocupaba lógicamente, que están las cosas, sobre todo ahora como para andar descuidándose en los aforos".

Esta persona subraya la normalidad del procedimiento que pone de relieve las buenas relaciones con la confesión minoritaria. Las dificultades parecen provenir más bien del tamaño de la población musulmana que potencialmente puede acudir al local y del espacio físico con el que cuenta esta comunidad, con el fin de evitar percances derivados de falta de aforo suficiente, tal y como parece que se habían procedido en otros lugares.

\section{Consideraciones finales}

En conclusión, en primer lugar nos gustaría resaltar la presencia de confesiones minoritarias en la CAPV como una realidad con un alto grado de implantación, tanto en términos de temporalidad como de implantación territorial. La mayoría de estas entidades religiosas han puesto de relieve una vocación de reconocimiento como agrupaciones de raíz religioso, ya que la mayoría están inscritas en el Registro de Entidades Religiosas del Ministerio de Justicia y tienen un alto grado de adhesión a las entidades federativas que les da acceso a desarrollar parte del contenido de los acuerdos que algunas de ellas tienen firmados con el Estado.

Sin embargo, a pesar de esta implantación, se destaca un relativo desconocimiento del hecho religioso no católico (confesiones minoritarias) en el conjunto de la sociedad vasca y de la existencia de posturas ambivalentes ante él mismo en cuanto a facilidades existentes para las prácticas de esas entidades, la valoración global de estas y la percepción de su presencia real en términos cuantitativos. La imagen de las confesiones religiosas minoritarias presenta aspectos de ambigüedad en los indicadores que hacen referencia al conocimiento y valoración de su presencia social, así como en la percepción sobre las facilidades que encuentran para sus prácticas religiosas, entre ellas la cuestión de los centros de culto.

La cuestión de los lugares de culto constituye un asunto altamente sensible para las minorías religiosas. La regulación normativa es lo suficientemente amplia y ambigua como para dar cabida a prácticas muy diferentes, algunas de ellas 
con tendencia discriminatoria y otras más flexibles e integradoras. El vacío legal existente en cuanto al otorgamiento de licencias posibilita el uso de criterios y decisiones contradictorias por parte de las Administraciones Públicas locales según los lugares y el caso, lo que se convierte en una considerable fuente de malestar. En cualquier caso, la disposición de espacios supone un requerimiento muy importante para la vida y desarrollo de los grupos religiosos.

Son bastantes las comunidades que tienen estrecheces a la hora de acoger a los participantes de la confesión religiosa dentro del local, y otras incluso no cuentan con locales de referencia. En el acceso a unos locales suficientes y adecuados se juega en parte la visibilidad que tiene un grupo dentro del espacio urbano. Desde una perspectiva de una sociedad que mayoritariamente está marcada por la presencia de la Iglesia católica y por el proceso secularizador que sitúa el fenómeno religioso en el ámbito de lo marginal y de lo privado, el local que pueda usar una minoría religiosa es clave para poder hacerse presente en la sociedad y demostrar tanto su existencia como la pluralidad que subyace en el tejido. 


\section{Referencias bibliográficas}

CONTRERAS MAZARIO, J.M. (2007): “La libertad de conciencia y convicción en el sistema constitucional español", Revista Cidob d'afers internacionals, 77, pp. 41-63.

DIEZ DE VELASCO, F. (2010): "The Visibilization of Religious Minorities in Spain”, Social Compass 57 (2), pp. 235-252.

GABINETE DE PROSPECCIÓN SOCIOLÓGICA (2012): Estudio Monográfico sobre Diversidad Religiosa, Vitoria-Gasteiz, Gobierno Vasco.

GARCÍA, D. Y URIARTE, L. (2011): “Las minorías religiosas y los medios de comunicación en la CAPV”, en RUIZ VIEYTEZ, E. (dir.), La diversidad religiosa en el País Vasco. Nuevos retos sociales y culturales para las políticas públicas, Bilbao, Deustuko Unibertsitatea.

MORONDO TARAMUNDI, D. y RUIZ VIEYTEZ, E.J. (eds.) (2014): Diversidad religiosa, integración social y acomodos. Un análisis desde la realidad local en el caso vasco, Bruselas, Peter Lang.
PÉREZ-AGOTE, A. (2012): Cambio religioso en España: los avatares de la secularización, Madrid, CIS.

PONCE, J. y CABANILLAS, J.A. (2011): Lugares de culto, ciudades y urbanismo. Guía de apoyo a la gestión de la diversidad religiosa, Madrid, Observatorio del Pluralismo Religioso en España.

RUIZ VIEYTEZ, E. J. (2013): “Derechos humanos, discriminación y diversidad religiosa en el País Vasco: la polémica sobre la apertura de centros de culto en Bilbao", MORENO, G. (ed.), Anuario Ikuspegi 2013, Bilbao, Ikuspegi, pp. 283-294.

RUIZ VIEYTEZ, E.J. (dir.); Pluralidades latentes. Minorías religiosas en el País Vasco, Barcelona, Icaria.

URRUTIA ASUA, G. (2016); Minorías religiosas y derechos humanos: Reconocimiento social y la gestión pública del pluralismo religioso en el País Vasco, Madrid, Akal. 